\title{
Mécanismes d'identification linguistique et jeunesse urbaine à Port-Vila (Vanuatu) : une approche anthropologique
}

\section{Leslie Vandeputte Tavo}

\section{(2) OpenEdition Journals}

Édition électronique

URL : https://journals.openedition.org/jso/6417

DOI : $10.4000 /$ jso. 6417

ISSN : 1760-7256

Éditeur

Société des océanistes

\section{Édition imprimée}

Date de publication : 15 décembre 2011

Pagination : 241-254

ISBN : 978-2-85430-037-4

ISSN : 0300-953x

\section{Référence électronique}

Leslie Vandeputte Tavo, « Mécanismes d'identification linguistique et jeunesse urbaine à Port-Vila (Vanuatu) : une approche anthropologique », Journal de la Société des Océanistes [En ligne], 133 | 2e semestre 2011, mis en ligne le 31 décembre 2014, consulté le 21 septembre 2021. URL : http:// journals.openedition.org/jso/6417; DOI : https://doi.org/10.4000/jso.6417

\section{c) () $(9)$}

Journal de la société des océanistes est mis à disposition selon les termes de la Licence Creative Commons Attribution - Pas d'Utilisation Commerciale - Pas de Modification 4.0 International. 


\title{
Mécanismes d'identification linguistique et jeunesse urbaine à Port-Vila (Vanuatu) : une approche anthropologique
}

par

\author{
Leslie VANDEPUTTE TAVO*
}

\section{RÉSUMÉ}

Port-Vila, capitale du Vanuatu, est le théâtre de profondes mutations culturelles et sociales, particulièrement visibles chez les nouvelles générations urbaines. Le pidgin bichlamar ${ }^{1}$, langue nationale de la République du Vanuatu depuis l'indépendance du pays en 1980, apparaît comme un outil essentiel à l'observation et à la compréhension de ces changements rapides et profonds, notamment dans le contexte de la capitale Port-Vila. Dans cet article, nous examinons les processus d'adaptation linguistique, d'innovation culturelle et d'identification collective que rend possible la créolisation $d u$ bichlamar, au sein d'une jeunesse urbanisée. Le bichlamar nous servira d'outil (à travers des mots, expressions, structures de phrases) pour analyser et mesurer les changements sociologiques en cours et l'accentuation des inégalités sociales grandissantes.

Mots-CLÉs : bichlamar, pidgin, ethno-linguistique, identité urbaine, Port-Vila, Vanuatu

L'expansion urbaine dans les archipels mélanésiens s'accompagne de nombreuses mutations (économiques, linguistiques, culturelles, sociales). Le Vanuatu ne fait pas exception, et Jean-Michel Charpentier soulignait déjà une " urbanisation rapide et anarchique " au début des années soixante-dix entraînée par un exode

\section{ABSTRACT}

Port Vila, the capital of Vanuatu, is a theatre of profound cultural and social mutations visible most particularly in the younger, urban generations. The pidgin bislama, the national language of the Republic of Vanuatu since its independence in 1980, appears to be essential in the observation and comprehension of rapid and, at times, strong changes in a context of breaking with the rural world. Within this article, we propose to examine the process of linguistic adaptation as a cultural innovation amongst the urban youth, as well as the collective identification with bislama today that has evolved into a creole mother-tongue. Bislama serves as a tool (through words, expressions and sentence structures) in the analysis and measure of current sociological changes, and the accentuation of growing social inequalities.

KeYwords: bislama, pidgin, ethnolinguistics, urban identity, Port-Vila, Vanuatu

rural massif (Charpentier, 1979 : 130). Les migrations circulaires ${ }^{2}$ largement majoritaires dans les années 1960 et 1970 ont laissé place à partir de l'indépendance à des migrations définitives (Siméoni, 2009 : 265).

Depuis l'indépendance, la croissance urbaine n'est plus seulement liée à l'exode des populations

1. Nous choisissons d'orthographier le pidgin bichlamar selon la graphie adoptée à l'indépendance mais il existe diverses autres façons de nommer ce pidgin : bislama, bichelamar, beach-la-mar, etc.

2. Les Ni-Vanuatu viennent à Port-Vila pour de courts séjours (entre un mois et un an) et pour des raisons économiques.

Doctorante EHESS-CREDO, leslievdp@gmail.com 
rurales mélanésiennes, mais également à l'arrivée d'investisseurs étrangers et de commerçants (australiens, néo-zélandais, chinois, indo-fidjiens) venus s'installer dans le pays. Tout cela rend plus complexe la situation linguistique et culturelle. Le Vanuatu fait partie des pays ayant la plus forte densité linguistique au monde (Moyse-Faurie, 2000) : aux 106 langues vernaculaires présentes dans l'archipel (Crowley, 2000) s'ajoutent, sans compter les langues asiatiques ou celles d'autres îles du Pacifique, les trois langues officielles que sont l'anglais, le français - les deux langues écrites issues de la colonisation - et le bichlamar, pidgin extensif ${ }^{3}$ servant de langue nationale et véhiculaire aux habitants.

Parler de la jeunesse au Vanuatu relève du pléonasme. En effet, la moyenne d'âge de la population du pays est de vingt ans et demi, d'après le National Census of Population and Housing de 2009. Cette jeunesse emblématise un ensemble de transformations culturelles et linguistiques. Dans cet article, nous nous proposons d'étudier les rapports entre la jeunesse urbaine de Port-Vila et le pidgin bichlamar devenu créole pour une grande partie de la population citadine. Ce dernier cristallise, à travers des registres de langue, des expressions, un lexique particulier et, plus que toute autre langue de l'archipel, les changements des représentations culturelles et l'évolution des modes de vie. Une analyse de ce pidgin en contexte urbain nous permettra d'identifier les mutations en cours chez les jeunes générations. Pour mener cette étude, nous définirons au préalable la jeunesse de Port-Vila en tant que catégorie sociologique et envisagerons le cadre dans lequel elle évolue. Puis nous évaluerons les relations entretenues entre les jeunes urbains et le pidgin bichlamar, les phénomènes d'appropriation de la langue et l'évolution du bichlamar urbain (notamment par rapport au bichlamar rural).

\section{Jeunesses mélanésiennes contemporaines en milieu urbain, l'exemple de Port-Vila}

\section{Port-Vila, capitale mélanésienne}

Port-Vila constitue le plus grand centre urbain du Vanuatu avec une population d'environ 44000 habitants (d'après le National Population and Housing Census (NPHC) de $2009 \mathrm{du} \mathrm{Va}$ nuatu National Statistics Office $\left.{ }^{4}\right)$. La capitale fut créée et investie par les Européens (investisseurs privés, planteurs ou missionnaires) à la fin du $\mathrm{XIX}^{\mathrm{e}}$ siècle et, à cette époque, s'apparente plus à un comptoir qu'à une ville. En 1906, la baie de Port-Vila devint le centre du condominium franco-britannique des Nouvelles-Hébrides. Les colons y établirent leurs structures administratives, mais la capitale ne prendra toutefois des allures de petite ville qu'à partir de 1942, pendant la Seconde Guerre mondiale, avec l'arrivée des troupes américaines à Efate. Les forces américaines mettent en place une infrastructure urbaine : piste d'aviation, routes, entrepôts portuaires, camps militaires - qui ont conservé leurs noms en bichlamar, Nambatu, Nambatri, toujours utilisés de nos jours pour désigner des quartiers de la ville -, hôpital (Siméoni, 2009 : 250). La croissance démographique urbaine de Port-Vila débuta réellement en 1955 et se poursuit toujours aujourd'hui. Les migrations mélanésiennes, qui se multiplièrent jusque dans les années 1970, sont qualifiées de circulaires (voir note 2). Dans la décennie qui précéda l'indépendance (juillet 1980), une rupture s'opéra : les migrations circulaires et temporaires eurent tendance à devenir permanentes (Bonnemaison, 1977). Plus que l'attraction pour le monde urbain, c'est le contexte rural qui explique l'évolution des migrations intérieures : les cours mondiaux du coprah, le développement de la scolarisation, les cyclones successifs (Bonnemaison, $1974: 260$ ) et les pénuries qu'ils engendrent du fait de la pression démographique. Après l'indépendance, la morphologie urbaine se modifia radicalement en raison d'une croissance démographique importante - le taux de natalité très élevé entraîna un accroissement naturel de $39 \%$ entre 1979 et 1989 (Siméoni, 2009 : 254) - couplé avec un solde migratoire interne toujours aussi important. La ville s'étendit et se déploya au nord (Anabru, Olhen, Freshwota, Tagabé) où il existait déjà une implantation mélanésienne ancienne (Bonnemaison, 1974). Durant les deux dernières décennies, Port-Vila a continué de croître de façon anarchique, sans plan d'urbanisme, ce qui accentua la ségrégation spatiale héritée du condominium des Nouvelles-Hébrides. Il existe une division nord-sud très marquée ; au sud et à l'est se trouvent les communautés aisées (étrangères mais aussi de plus en plus ni-vanuatu) alors quau nord les quartiers sont fortement " bidonvillisés ». Aujourd'hui, la croissance urbaine est toujours aussi forte avec une augmentation de $4 \%$

\footnotetext{
3. Un pidgin se distingue d'un créole selon des critères linguistiques : il comporte une grammaire, une syntaxe, un lexique jugé moins développé et moins complexe qu'un créole. Le pidgin extensif en revanche est linguistiquement similaire au créole, mais il se différencie du pidgin d'un point de vue sociologique : il n'est pas la langue maternelle des individus. À l'inverse, lorsqu'une langue de contact est désignée comme étant un créole cela signifie qu'il s'agit de la langue maternelle des individus (Mühlhäusler, 1986 ; Keesing, 1988 ; Holm, 2000 ; Siegel, 2008).

4. Luganville, situé sur l'île d'Espiritu Santo, est la deuxième ville du pays avec une population d'environ 13000 habitants (NPHC de 2009).
} 


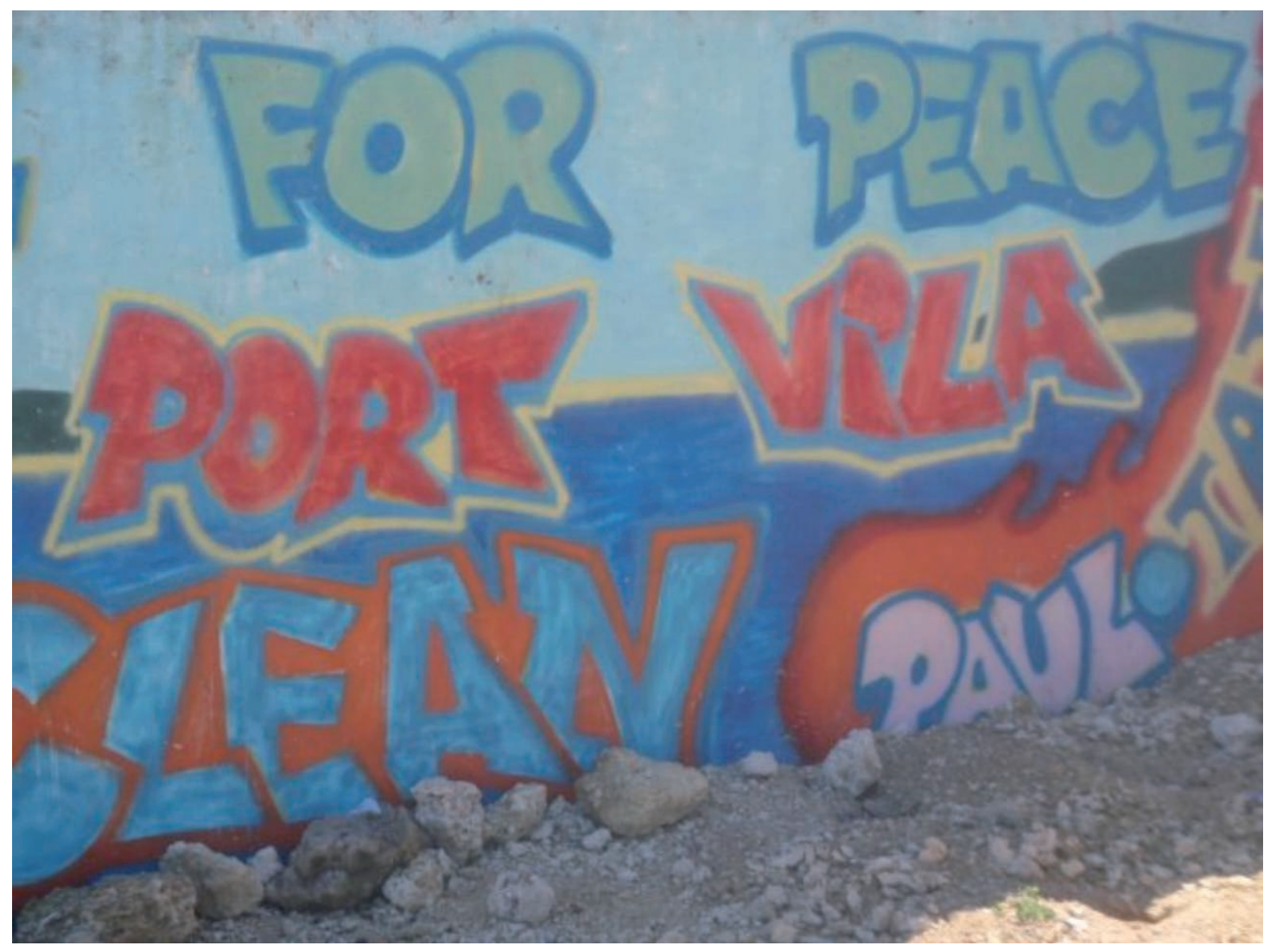

Рното 1. - Nouvelle art de rue en développement dans la capitale. Tag situé derrière le marché sur le front de mer (cliché de l'auteur, avril 2009)

par an (concernant Port-Vila, NPHC de 2009). La centralisation de l'État et la concentration des administrations dans la capitale renforcent l'hégémonie de la ville face au faible poids démographique et politique que joue le reste de l'archipel, à l'exception de Santo. Bien qu'il existe aujourd'hui une volonté de renforcer le pouvoir des provinces ${ }^{5}$, la situation reste très inégalitaire et l'attrait pour la capitale inchangé, voire renforcé. Avec une croissance démographique de 3,5\% par an, l'archipel tout comme la capitale doivent faire face à de nouveaux défis (infrastructure, santé, éducation, etc.).

\section{Jeunesse urbaine contemporaine}

Dans cette étude, nous portons notre attention sur les jeunes urbains de Port-Vila, des deux sexes, appartenant aux classes d'âge de 15 à 25 ans, scolarisés, employés ou en recherche d'emploi et ayant grandi dans la capitale. Aujourd'hui, il s'agit là de la deuxième génération d'urbains ayant rompu le contact avec le monde rural (à l'inverse des migrations circulaires des années 1950 à 1970 décrites supra). Cette population porte en elle les inégalités socio-économiques de l'ensemble de la société urbaine ni-vanuatu. Nous distinguons différentes catégories d'individus : scolarisés, employés, chômeurs recherchant ou non un emploi.

Aucune statistique spécifique concernant les jeunes de 15 à 25 ans ne permet de dire combien d'entre eux sont scolarisés. En revanche, nous savons que $5 \%$ de la population de plus de 15 ans est analphabète et que seulement $7 \%$ poursuit ou a poursuivi des études dans le supérieur (NPHC de 2009). Il semble que peu nombreux sont ceux qui ont accès à des études au-delà de la douzième (classe correspondant à la seconde dans le système français, qui est considéré comme un niveau relativement élevé $\left.{ }^{6}\right)$. De plus, les statistiques concernant l'éducation apportent assez peu d'informations fiables, car de nombreux éléments ne sont pas pris en compte : interruptions en cours d'année (pour non-paiement de

5. L'archipel est découpé administrativement en six provinces distinctes, qui sont des acronymes : Tafea au sud (Tanna, Futuna, Erromango, Anatom et Aniwa), Shefa où se trouvent Port-Vila (Shepherd, Efate, Epi et Emae), Malampa (Malekula, Ambrym et Paama), Penama (Pentecôte, Ambae et Maewo), Sanma (Santo et Malo) et Torba (Torres et Banks) au nord.

6. La douzième permet par exemple de s'inscrire à l'Institut de formation des enseignants du Vanuatu (IFEv), de participer au concours d'entrée aux écoles d'infirmière ou de police, ou encore d'aller à l'Université du Pacifique Sud (moyennant des équivalences). 
frais de scolarité, grossesse, etc.), absentéisme répété, etc. Port-Vila concentre de nombreuses activités totalement absentes ailleurs dans l'archipel. Cet attrait économique - la recherche d'un emploi rémunéré - fait partie des principales raisons expliquant l'exode rural. Mais rares sont ceux qui peuvent accéder à un emploi, souvent par manque de qualification; ainsi, en zone urbaine, presque $12 \%$ de la population n'a pas de travail. Ce constat touche majoritairement les jeunes puisqu'un chômeur sur deux a moins de vingt-cinq ans (Siméoni, 2009). Bonnemaison distinguait dans les années 1970 trois catégories sociales basées sur l'emploi :

- la plus basse couche regroupe les manœuvres et ouvriers non qualifiés ;

- la classe intermédiaire est constituée d'ouvriers spécialisés et qualifiés et de petits entrepreneurs ;

- on voit également émerger une classe moyenne, voire bourgeoise, composée de fonctionnaires, techniciens ou agents hautement qualifiés (Bonnemaison, 1977 : 31-34).

Cette description correspond à la société pré-indépendance des Nouvelles-Hébrides ; aujourd'hui la situation a quelque peu évolué. La classe moyenne actuelle s'est développée, les salaires ont augmenté et ont permis de donner un réel pouvoir d'achat à cette classe moyenne plus favorisée. Une catégorie intermédiaire a vu le jour, qui englobe majoritairement les moins de 30 ans n'ayant pas d'emploi fixe mais susceptibles d'effectuer de petits travaux épisodiques rémunérés. Ces jeunes se qualifient eux même de SPR, sigle qui veut dire Sperem Pablik Rod en bichlamar - "traîner dans la rue " en français. Dans son article Killing Time in a Postcolonial Town: Young People and Settlements in Port-Vila, Vanuatu, Mitchell s'attache à décrire les comportements de ce lumpenproletariat (2004 : 364). Un film documentaire intitulé Kilim Taem fut également réalisé par Mitchell et le Vanuatu Young People Project (vypP $)^{7}$. Certaines aspirations et difficultés de cette nouvelle génération de jeunes urbains y sont mises en avant. Nombre d'entre eux ont vécu toute leur vie à Port-Vila, ont arrêté leur scolarité très tôt et n'ont pas d'autres occupations que de " traîner entre potes ${ }^{8}$ ». Les expressions synonymes kilim taem ou westem taem signifient littéralement " tuer le temps " et " perdre son temps "; elles expriment parfaitement la représentation que ces jeunes urbains se font de leur quotidien. Dans ce film, ils commentent leur réalité quotidienne : regarder des vidéos, boire du kava, consommer de l'alcool, sortir en boîte de nuit, se bagarrer, voler, fumer des cigarettes, discuter, jouer de la guitare et, souvent, ils affirment n'avoir aucun espoir de trouver du travail. Éloignés des réalités des " îles" ", ils idéalisent le mode de vie rural tout en ayant conscience qu'ils ne pourraient pas aller y vivre (sans attaches familiales fortes, sans biens fonciers, avec des problèmes d'adaptation). Le manque de confiance dans le système politique et dans ses représentants accentue le sentiment d'abandon de ces jeunes en perte de repères culturels et sociaux ${ }^{10}$. La mixité ethnique des individus participe de ce phénomène; en zone urbaine, le regroupement communautaire entre populations originaires des îles extérieures, observé durant les premières vagues de migration circulaire, a laissé place à une mixité de plus en plus forte. Les enfants issus de mariages interethniques grandissant à Port-Vila ne connaissent généralement pas l'île natale de leurs parents et sont par conséquent coupés des coutumes et du mode de vie insulaire rural, tout comme de la langue vernaculaire. Pour un grand nombre de jeunes urbains, le bichlamar s'est imposé comme langue maternelle. Voyons maintenant les phénomènes d'appropriation identitaire entre la jeunesse créolisée de PortVila et la langue nationale du Vanuatu.

\section{Innovations urbaines et ajustements linguis- tiques du bichlamar}

\section{Omniprésence du bichlamar en milieu urbain}

À Port-Vila, la situation linguistique est avant tout caractérisée par sa complexité, comme nous l'avons rappelé en introduction. Le Vanuatu dispose d'une grande richesse linguistique à laquelle se sont ajoutées les langues des migrations externes. Après l'installation initiale des colons européens, accompagnée de quelques familles de travailleurs kanak, les communautés asia-

7. Le vypr fut mis en place en 1997 par Jean Mitchell et intégré au Centre culturel du Vanuatu (vKs). Il s'agit d'un projet ayant pour but de faire parler les jeunesses urbaines de Port-Vila. Ce projet a été financé par le gouvernement du Vanuatu, AuSAID, Save the Children Australia et l'Unicef. La vidéo Kilim Taem reprend des recherches réalisées auprès de jeunes personnes; elle a été financée par l'UNICEF et AUSAID et réalisée en partie par l'équipe du vKs, dont Jacob Kapere (conservateur en chef du département audiovisuel du Centre culturel du Vanuatu).

8. Expression familière traduite directement d'une interview issue de la vidéo Kilim Taem dans laquelle un jeune homme décrit son quotidien par l'expression en bichlamar wobaot wetem ol fren.

9. Les « ̂̂les » désignent communément tout ce qui est en dehors de Port-Vila.

10. Régulièrement les journaux dénoncent la corruption connue et critiquée ; récemment, des groupes de musique reggae ont écrit sur le manque de responsabilité des politiciens - Youth Life "Give us equal rights" - ; pourtant la situation reste inchangée. 
tiques font partie des plus anciennes migrations (vietnamiennes et chinoises ${ }^{11}$ principalement), suivies par celle des Wallisiens installés pour la plupart depuis les années soixante ${ }^{12}$ et, enfin, les Indo-Fidjiens arrivés plus récemment ${ }^{13}$. L'utilisation du bichlamar est renforcée par le fait que Port-Vila ne regroupe pas ou peu de locuteurs d'une même langue dans un même quartier : ce constat a fait l'objet d'un travail personnel d'enquête de terrain lors d'un projet consistant à élaborer une carte sociolinguistique de la capitale. Cette étude s'est finalement révélée non pertinente en raison d'une trop grande mixité dans les quartiers de FreshWota wan, FreshWota tu, FreshWota foa et Nambatri où l'enquête a été réalisée (septembre-décembre 2009). Certes, il existe des lieux dans la capitale où une communauté d'une même île se trouve majoritaire comme, par exemple, à Seaside Tongoa ou à Blacksand, mais, aujourd'hui, la majorité des quartiers regroupent des communautés d'îles différentes. La plus grande partie des échanges linguistiques se pratiquent en bichlamar ; $85 \%$ de la population résidant en milieu urbain affirment maîtriser cette langue, contre $75 \%$ pratiquant une langue vernaculaire (National Population and Housing Census, 2009). Charpentier assure qu'à la fin des années 1970, l'ensemble de l'archipel comptait $6 \%$ de personnes créolisées. Malgré l'absence de chiffres, nous pouvons affirmer qu'aujourd'hui, à Port-Vila, les individus parlant le bichlamar comme langue maternelle sont en forte augmentation en raison du nombre toujours croissant de mariages interethniques, de la mixité de plus en plus grande dans les zones urbaines et périurbaines, et de l'environnement "bichlamarisé " favorisant l'apprentissage du pidgin au détriment des langues vernaculaires. Au sein des communautés (ou simplement des familles) utilisant une langue vernaculaire, nous pouvons défendre (après de nombreuses enquêtes réalisées dans les quartiers de FreshWota wan, FreshWota tu et d'Anabru entre 2008 et 2010) que son usage est fortement influencé par le bichlamar. L'omniprésence du bichlamar favorise son insertion dans les langues vernaculaires. Il va souvent être préféré par les locuteurs dans l'emploi d'expressions idiomatiques :

\footnotetext{
i stret: "c'est bon ", "c'est d'accord "

ale: " allons-y, vas-y ", "puis » ou bien par «d'accord " hemia nao : expression figée signifiant "voilà » ou «c’est ça ».
}

De chiffres, de jours de la semaine, de mois :

wan handred, wan taosen, etc. : mille, cent mondae, tesde, sarede, etc. : lundi, jeudi, samedi maj, julae, oxis, oktoba, etc. : mars, juillet, aout, octobre.

De mots renvoyant à un lexique " moderne » :

mobael désignant le téléphone portable, plen : avion,

komputa ou laptop: ordinateur et ordinateur portable.

Toutes ces catégories n'ont pas forcément d'équivalent dans les langues vernaculaires (par exemple, le terme " mois " se dit dans la langue de Port-Sandwich, au sud Malekula, paci qui signifie la lune, mais il n'existe pas de mot correspondant aux douze mois du calendrier grégorien).

\section{Multiplication des échanges, développement des transports et outils de communication comme fac- teur de renforcement du pidgin}

La transformation du pidgin, entraînée par le développement des médias et des transports, favorise son extension. En 1966, la première radio est créée au Vanuatu, appelée Radio Vila, média du condominium qui diffuse dans les trois langues officielles. Les émissions en bichlamar font l'objet d'une très forte audience (Charpentier, 1979). En utilisant le pidgin, Radio Vila va conférer à cette langue un rôle unificateur, car contrairement à l'anglais ou au français, le bichlamar n'est pas un vecteur de division au sein de la population. Par ailleurs, son utilisation sur un média de diffusion nationale permet d'harmoniser et de populariser le pidgin (Tabani, 2002 : 258-259). En 1978, Radio Vila est remplacée par le Service de radio diffusion des Nouvelles-Hébrides et s'étend à l'ensemble - ou presque - des îles du Vanuatu. Aujourd'hui, Radio Vanuatu couvre tout l'archipel, avec plus de $70 \%$ des émissions en bichlamar (Siméoni, 2009 : 339). En zone urbaine, il existe d'autres radios commerciales comme Tropical FM ou encore Capitol FM107 (créée en 2007) qui émettent dans les trois langues et majoritairement en bichlamar. La télévision fut introduite au Vanuatu en 1991 et ne diffuse qu'à Port-Vila et en zone péri-urbaine. Au départ, la majorité des émissions (environ trois à quatre heures par jour) étaient en français ou en anglais ; aujourd'hui, le bichlamar reste la langue privilégiée dans la diffusion de l'information : journal, émissions musicales, reportages

11. Les Vietnamiens sont arrivés depuis la Province du Tonkin - dans le nord du Vietnam - au début des années 1920. Les migrations chinoises sont plus diffuses car les communautés sont arrivées progressivement après la Seconde Guerre mondiale puis plus régulièrement jusqu'à nos jours. Les Vietnamiens se sont installés dans différents quartiers de Port-Vila (plutôt au nord de la ville) alors que les Chinois sont restés dans le centre-ville, habitant des petits appartements au-dessus de leurs magasins (Bonnemaison, 1977).

12. La migration wallisienne correspond à une demande de main-d'œuvre dans les années soixante pour travailler dans la mine de Manganèse à Forari au nord d'Efate. À la fermeture de la mine dans les années quatre-vingts, la plupart est restée et s'est installée majoritairement dans le quartier de Nambatri à Port-Vila, sur un terrain que la mission catholique leur attribua (Bonnemaison, 1977).

13. Phénomène migratoire observé depuis seulement cinq ans. 
sur les coutumes des îles, pièces de théâtre et feuilletons Wan Smol Bag ${ }^{14}$, etc. sont en bichlamar. Cependant, la TBv (Television Broadcast Vanuatu) n'est pas entièrement en bichlamar, faute de budget, mais aussi pour garder une ouverture sur la Nouvelle-Calédonie, la France et l'Australie. De plus en plus, le bichlamar est utilisé dans des spots publicitaires des entreprises locales et étrangères - Au Bon Marché (supermarché), Digicel et TVL (entreprises de télécommunications), Wilco (fourniture pour le secteur du втр et la maison), Entreprise Traverso Sandrino (installation de stores anticycloniques), etc. En 2009, outre la création d'un nouveau journal hebdomadaire $^{15}$ The Vanuatu Times, publiant dans les trois langues officielles, une revue mensuelle Storian a fait son apparition. Storian associe des articles sur les spécificités de l'archipel - reportage mode sur les aeland dress ${ }^{16}$, interview d'hommes politiques, article sur les nouveaux stringbands ${ }^{17}$ en vogue - à d'autres mentionnant les tendances occidentales, l'actualité cinématographique et les nouvelles technologies (ordinateurs, téléphones cellulaires, caméras, appareils photo, consoles de jeux vidéo, baladeurs musicaux, etc.). Storian diffuse principalement en anglais ; toutefois, d'après les créateurs de cette revue, une volonté réelle existe pour développer l'usage du pidgin, considéré selon eux comme le principal vecteur de diffusion.

À travers les médias, la publicité joue aujourd'hui un rôle très important dans la diffusion du bichlamar. La publicité existait déjà avant l'indépendance. Elle fut introduite et développée pendant le condominium par les deux administrations coloniales du pays, les Britanniques et les Français (New Hebrides News, 1975, 1976). Toutefois, son usage restait limité à la presse locale et à destination d'un lectorat essentiellement non mélanésien (publicité pour des hôtels, de petites entreprises, etc.), puisqu'une majorité de la population locale du pays demeurait analphabète. Après l'indépendance et avec le développement des médias (radio, télévision), la publicité se développa lentement. Des spots de télévision aux campagnes d'affiches (phénomène apparu il y a trois ans, lors de l'implantation de l'entreprise de téléphonie Digicel qui a collé ses gigantesques affiches $-4 \mathrm{~m} \times 4 \mathrm{~m}$ - aux principaux carrefours de la ville), le Vanuatu possède désormais son lot de publicités pour des enseignes commerciales locales et internationales, surtout en milieu urbain (Luganville, Port-Vila). La multiplication des campagnes publicitaires utilisant le bichlamar dans les publicités renforce la crédibilité conférée au pidgin (Vandeputte Tavo, 2011).

En plus des médias, le développement des transports en bateau et l'augmentation de la desserte aérienne entre la capitale et les îles de l'archipel ont accentué la diffusion, voire l'uniformisation du pidgin. Le transport maritime de passagers s'est récemment développé avec l'arrivée de deux nouveaux catamarans, Big Sista (septembre 2010) et Efate Queen (février 2011), qui desservent les îles d'Epi, de Malekula, de Santo, d'Ambae et de Pentecôte. Le coût du service, moins élevé que le transport aérien, permet de se rendre plus aisément dans les îles (à l'occasion des fêtes de fin d'année notamment). Les autres bateaux reliant les îles entre elles sont des embarcations souvent bien plus vétustes transportant des cargaisons (vivres, coprah, matériaux, carburant, etc.), mais qui acceptent également des passagers selon la place disponible. Le Vanuatu dispose aussi d'une infrastructure aérienne relativement développée avec vingt-neuf aérodromes et pistes d'atterrissages répartis dans tout l'archipel, et une flotte appartenant à la compagnie nationale Air Vanuatu. L'utilisation du bichlamar progresse avec la multiplication des contacts et des échanges entre les îles et avec la capitale.

Laccès plus facile à différents modes de télécommunication change également progressivement les usages du pidgin. L'introduction de Digicel, en juin 2008, joua un rôle essentiel dans ce processus, en favorisant l'accessibilité au téléphone portable. En étendant une couverture réseau à l'ensemble de l'archipel, l'entreprise a contribué à réduire les distances et a démocratisé l'usage du téléphone portable. Aujourd'hui, la majorité des $\mathrm{Ni}$-Vanuatu disposent d'un téléphone mobile et restent en contact avec leurs proches qui ont migré à Port-Vila ou Luganville. Ce moindre isolement se traduit, sur le plan linguistique, par un usage de plus en plus important du bichlamar, sous forme d'expressions et de mots introduits dans la langue vernaculaire. Lutilisation grandissante de messages textuels transportés appelés text, mesej, text-mesej ou sms en bichlamar, participe à diffuser cette langue. Le bichlamar apparait comme l'idiome privilégié des échanges

14. L'association organise, à travers des bandes dessinées, des pièces de théâtre ou des sketches, des campagnes éducatives sur la santé, la protection de l'environnement, le développement du tourisme, etc. Ses projets sont financés par des bailleurs de fonds qui peuvent demander de traiter un sujet spécifique comme ce fut le cas pour le livret Yumi evriwan $i$ nidim blong save Maths, commandé par le Japan International Cooperation Agency (JICA).

15. La presse locale écrite est composée du quotidien bilingue (anglais, bichlamar) the Daily Post et de deux hebdomadaires trilingues (anglais, français, bichlamar), The Vanuatu Independent et le Vanuatu Times.

16. Aeland dress signifie littéralement " robe des îles». Il s'agit des robes dites mission imposées par les missionnaires et qui furent depuis adaptées aux couleurs locales. Elles sont caractérisées par un tissu aux motifs floraux et aux teintes vives, par leur longueur à mi-mollet et une coupe large couvrant la silhouette).

17. Stringband: "groupe composé en général de jeunes garçons, jouant des instruments à cordes (guitare et ukulélé). Cette forme de musique est née pendant la colonisation, elle comporte de fortes influences polynésiennes et modernes. Son développement est encouragé par le secteur touristique » (Stern, 2002 : 419). 
en messages instantanés ; d'une part, le caractère oral des langues vernaculaires limite leur utilisation écrite ; d'autre part, les messages textuels furent dans les premiers temps de la téléphonie utilisés uniquement par un public de jeunes urbains créolisés ou utilisant le bichlamar dans leurs échanges quotidiens. La particularité des messages textuels réside dans l'utilisation récurrente d'analogies sonores destinées à réduire le temps de rédaction et le coût du message (le nombre de caractère étant limité à 160) ; cette caractéristique n'est pas propre au bichlamar puisqu'elle existe dans la majorité - sinon la totalité - des échanges textuels. Ainsi les messages instantanés accentuent l'usage du pidgin sous une forme nouvelle :

Message : Ba mi go luk m lo taon, $z$ ?

Bichlamar : Bae mi go luk hem long taon, stret?

Traduction : Je vais le voir en ville, ok ?

M. : U2fala nmo i talm...

B. : Yutufala nomo i talem...

T. : Vous me dites...

M. : 1 m i no gud?

B. : Wanem i no gud?

T. : Qu'est ce qui ne va pas?

Ce phénomène participe de l'appropriation du pidgin par la jeunesse urbaine qui différencie son usage du pidgin. Le téléphone portable n'est pas ici le seul facteur d'un tel constat, mais participe activement, avec le développement des médias et des transports, à cette diffusion très importante. Cette situation ne s'observe pas partout mais dépend des zones de contact avec Port-Vila et les autres îles. Certaines régions d'une île sont fortement "bichlamarisées " comme par exemple les villages d'Okapa ou de Port-Olry du nord de Santo et ceux de Lamap ou Lakatoro à Malekula. Dans ces régions périphériques, le bichlamar remplace depuis plusieurs décennies des mots oubliés et perdus dans la langue locale (par exemple, à Port-Sandwich, le mot cuisine se dit aujourd'hui en bichlamar, kitchen). Dans des îles plus isolées, le bichlamar tend à s'immiscer de façon plus insidieuse avec l'utilisation d'un vocable standardisé, idéologiquement imposé à une échelle nationale comme kastom ${ }^{18}$ (voir Tabani, 2002).
L'environnement urbain change et les citadins participant à ces bouleversements évoluent avec lui. La jeunesse urbaine est au cœur de ces mutations. Des lieux de rassemblements ont vu le jour, tels que le Wan Smol Bag, une compagnie théâtrale créée en 1989 en tant qu'oNG (voir note 14), qui a conçu en 2005 un centre d'activité pour les jeunes, le Yut Senta, dans lequel ils peuvent y apprendre l'informatique, jouer de la musique, danser le hip-hop, organiser des groupes de réflexion sur des thématiques et problématiques variées. Le dynamisme et le changement des préoccupations des jeunes adultes participant aux activités du Yut Senta sont révélateurs des nouveaux types d'intérêts sur le plan des loisirs culturels. Lors des ateliers de réflexion organisés pour déterminer les prochaines thématiques d'une nouvelle bande dessinée ou pièce de théâtre, les débats ont illustré des réalités bien différentes de celles présentes en contexte rural ou de celles visibles il y a encore trente ans. Un groupe de discussion sur les problèmes liés à la sexualité a montré que les grossesses précoces sont une véritable source d'inquiétude; ces débats ont donné naissance à la bande dessinée $M i$ no wantem gat bel! ${ }^{19}$ qui met en scène une jeune femme abusée qui prend la pilule du lendemain pour éviter une grossesse. Un autre livret, Storian blong Rachel, Ol gel! No letem ol boe oli spolem laef blong $y u !^{20}$, raconte l'histoire d'une jeune fille qui tombe enceinte et explique les méthodes contraceptives ${ }^{21}$. D'autres bandes dessinées évoquent le respect des droits de la femme comme Gel Ambae wetem boe Tanna ${ }^{22}$ ou Storian blong Susan wetem Mika ${ }^{23}$ qui illustrent les problèmes de violence conjugale. Ces livrets éducatifs se focalisent sur les différences de genre et le désir d'émancipation des jeunes femmes en milieu urbain qui réclament plus de droits et de respect que dans le passé. Le Wan Smol Bag réalise aussi des campagnes de prévention contre le virus de l'immunodéficience humaine (VIH) qui touche principalement les villes (les dépistages n'étant pas systématiques, l'ampleur de diffusion du virus n'est pas encore connu : cinq cas officiels sont actuellement enregistrés). La bande dessinée intitulée Kleva $i$ save winim AIDS? ${ }^{24}$ est

18. À Mere Lava par exemple, dans les îles Banks, les individus utilisent le mot kastom au lieu des termes vernaculaires renvoyant aux usages de la coutume, mais sous une forme moins générique. Dans la majorité des langues vernaculaires, le terme kastom se traduit par une expression renvoyant aux "savoirs ancestraux ». Le mot kastom mis en avant dans les mouvements indépendantistes des années soixante-dix s'est imposé nationalement pour désigner un ensemble de pratiques coutumières et traditionnelles. Charpentier écrit que le terme kastom, « issu de l'anglais custom "coutume, usage, habitude", a un champ sémantique très large, beaucoup plus étendu que celui du terme anglais dont il est issu. Il signifie certes les habitudes, les usages, les coutumes ancestrales mais il fait référence également aux croyances, aux chants, aux danses, aux légendes, à l'ordre social existant encore " (Charpentier, 1979).

19. "Je ne veux pas être enceinte! ", ma traduction.

20. "L'histoire de Rachel. Les filles, ne laissez pas les garçons gâcher votre vie ! ", ma traduction.

21. Le Wan Smol Bag dispose également d'un planning familial où chacun peut avoir accès à la pilule contraceptive, à la pilule préventive et aux préservatifs. Il est également possible d'y faire des tests de détection du viH.

22. "La jeune fille d'Ambae et le garçon de Tanna ", ma traduction.

23. "L'histoire de Suzan et Mika ", ma traduction.

24. "Le guérisseur/ le magicien/ le clairvoyant peut-il guérir le sIDA ? ", ma traduction. 
caractéristique des questionnements de citadins confrontés à des situations inédites. Un kleva, issu de l'anglais clever " doué, intelligent ", est une personne capable de guérir, de soigner ou de protéger les individus contre tous les maux, généralement à l'aide de plantes médicinales ; en zone urbaine, les kleva demandent une rémunération pour leurs services. Ce livret fut donc créé en réponse à la " légende urbaine " de Port-Vila selon laquelle le siDA n'était pas une maladie incurable et qu'elle était soignable par les kleva. En 2007, Wan Smol Bag a rencontré des difficultés avec le Conseil national des chefs (Malvatumaori) qui accusait la compagnie théâtrale d'«aborder des sujets sensibles et "interdits" (tabous) de façon trop libérée $e^{25}$ "; pourtant la fréquentation et le dynamisme de l'association et du Yut Senta montrent bien une envie et un besoin de parler de sujets jusque-là occultés.

\section{Langues de contact et identifications collectives en milieu urbain}

\section{Créations lexicales}

À cette nouvelle culture urbaine correspondent de nouvelles formes d'identification collective. Lenvironnement urbain est incompatible avec de nombreuses pratiques relevant du champ de la kastom. Les savoirs traditionnels ne disposent guère d'ancrage dans l'expérience quotidienne des gens vivant en ville. Ces changements culturels influencent largement un bouleversement linguistique du bichlamar, quand bien même celle-ci demeure toujours apparentée à la catégorie des langues de contact. Pour mémoire, une langue de contact (lingua franca, sabir, pidgin, créole) est définie par le caractère historiquement récent de son invention et de sa diffusion, par la difficulté à lui attribuer une origine précise et par sa très grande instabilité. Les jargons, pidgins et autres créoles sont beaucoup plus sensibles aux changements sociaux et aux adaptations culturelles que les langues plus anciennement établies et codifiées. Les transformations du contexte socio-économique et les changements dans les représentations culturelles inter-réagissent de manière beaucoup plus immédiate avec les innovations de vocabulaire et de grammaire. Les langues de contact s'insèrent dans ce qui est convenu d'appeler un continuum linguistique. Si les interactions entre les individus persistent, un sabir, jargon trade en anglais, exclusivement réservé à des opérations commerciales et doté d'un lexique limité et d'une grammaire embryonnaire, évolue ensuite en pidgin puis en pidgin extensif, comme c'est le cas du bichlamar. Lorsque cette langue de contact devient la langue première des locuteurs, dite maternelle, on parle alors de créole. De nouveaux mots ou de nouvelles expressions sont créés pour exprimer des pratiques inédites liées aux loisirs, à l'usage de drogues ${ }^{26}$, au sexe, etc. Des nouvelles locutions sont utilisées pour décrire des états d'ébriété, par exemple pour dire qu'une personne est sous l'emprise du cannabis :

$$
\begin{aligned}
& \text { man ia } \text { i bun : "ce mec est déchiré ", } \\
& \text { man ia i laet : " ce mec est allumé ". }
\end{aligned}
$$

Dans les années 1990, Crowley répertoriait les différentes expressions décrivant les effets du kava, suite à la commercialisation du breuvage dans la capitale ; certaines d'entre elles s'entendent encore aujourd'hui, pour désigner une personne enivrée par le kava et ayant du mal à se déplacer :

$$
\begin{aligned}
& \text { fowil: « marcher à quatre pattes ", } \\
& \text { dakdak: « canard ». }
\end{aligned}
$$

Pour désigner la couleur du kava qui s'apparente à de la boue, on dira :

wota blong pig: «l'eau des cochons ».

On emploiera un terme dérivé de la boxe - nokaot, " frapper " - pour exprimer la force inattendue du kava ; il est ainsi fréquent d'entendre quelqu'un dire :

$$
\text { kava i nokaotem mi : "le kava m'a mis } \mathrm{KO} \text { ». }
$$

En revanche, d'autres expressions notées par Crowley ont complètement disparu comme :

woklet : «se réveiller tard " (dérivé de l'anglais work late) cette expression désigne un kava dont l'absorption provoque des difficultés pour se réveiller le lendemain); neskava, dérivé du bichlamar neskafe qui désigne de façon générique le café instantané, le neskava était un projet de commercialisation du kava sous forme de poudre soluble à laquelle il suffisait d'ajouter de l'eau pour obtenir le breuvage ;

25. Informations recueillies lors d'un entretien avec Jo Dorras, la rédactrice des publications, le 24 mars 2011.

26. Les drogues - ou substances altératrices de conscience - présentes au Vanuatu sont : le kava (boisson ancestrale consommée initialement lors de cérémonies, connue pour ses effets relaxants et anesthésiants, il fut commercialisé et industrialisé à la fin des années quatre-vingts. Aujourd'hui, Port-Vila compte de trois à quatre cents kava-bars), l'alcool (la boisson la plus consommée est la bière Tusker; il existe aussi une fabrication artisanale appelée umbro ou drae pam, issue de la fermentation de fruits), et le cannabis, qui a fait son apparition il y a une dizaine d'années et se développe fortement. Il est associé à une culture rasta/jamaïcaine très populaire chez les jeunes. Sa consommation augmente en raison de son faible coût, même si les tribunaux sanctionnent parfois cette pratique pour l'exemple. Il n'y a pas de réelle poursuite pénale, comme en témoigne la confiscation des cigarettes "parfumées » lors du concert de Kimani Marley qui a fait la Une du quotidien Daily post le lendemain). 
kano, désigne un kava très fort ; le terme kano vient du français et son usage en bichlamar renvoie à plusieurs sens du mot : d'une part, il désigne la pièce d'artillerie envoyant des projectiles ; d'autre part, il renvoie à l'usage familier exclamatif du mot canon signifiant fantastique, super (Crowley, 1992).

Ces expressions disparues ont aujourd'hui laissé place à de nouvelles:

waes blong kava i kam: "l'effet du kava monte ",

kava i rekem mi / kava i sakem mi : « le kava me fait beaucoup d'effet ",

nakato : " bernard-l'hermite (se dit d'une personne grisée par le kava, qui marche très lentement).

En plus d'avoir un lexique très riche renvoyant aux effets du kava en lui-même, les jeunes urbains utilisent aussi beaucoup de formules codées pour ne pas dire directement qu'ils vont au kava-bar :

Mi wantem go long postofis: "Je veux aller à la poste", Mi go luk olfala woman antap: "Je vais voir ma mère - la vieille femme - là-haut",

Mi go chekem laet nomo : «Je vais juste vérifier si la lumière est allumée "; une petite lumière - lampe électrique ou lampe kérosène dite " tempête " - indique toujours s'il y a ou non du kava dans les nakamal ${ }^{27}$.

Ils peuvent choisir ces expressions détournées parce qu'ils n'ont pas le droit de s'y rendre - l'âge auquel il est possible de boire du kava peut varier selon les individus et les régions dont ils sont originaires, le critère pouvant être la poussée de la barbe ou la circoncision - ou parce qu'ils n'ont pas les moyens d'inviter des personnes à venir avec eux ou simplement par humour.

Les jeunes de Port-Vila sont les plus créatifs et les plus productifs lexicalement, en raison de leur utilisation quotidienne de la langue nationale. Ils développent un slang bichlamar ou un bichlamar des rues, forme du pidgin utilisant beaucoup d'expressions comprises principalement par eux et souvent inventées à partir d'anecdotes. On observe une double utilisation du bichlamar comme marqueur identitaire. D'une part, le bichlamar est utilisé comme une façon de se démarquer des autres générations : une appropriation très forte du pidgin est perceptible chez ces jeunes qui sont en rupture avec la langue vernaculaire de leurs parents. Charpentier faisait déjà remarquer à la fin des années soixante-dix que les personnes âgées qualifiaient le bichlamar de lanwis blong rod qui signifie " langue bâtarde " (pikinini blong rod est une expression qui se traduit en bichlamar par " enfant bâtard ") ; alors que les jeunes décrivent le pidgin comme lanwis blong mifala qui veut dire "notre langue" (Charpentier, 1979). Une distinction générationnelle est perceptible dans le jugement des locuteurs sur le bichlamar, qui s'est aujourd'hui accentuée. Par ailleurs, au sein de cette même jeunesse, le bichlamar comporte des variantes selon les individus, leur appartenance sociale et leur occupation (scolarisé, employé). Les jeunes de Port-Vila se réunissent par groupe dans des squats au sein des différents quartiers de la ville. Le terme squat est une nouvelle entrée lexicale ; elle n'est pas répertoriée dans le dictionnaire de Crowley (1995). Son sens differe de l'acception qu'il a en français puisqu'il désigne ici un lieu occupé en toute légalité :

Hem i stap wetem ol squat blong hem "Il est avec sa bande ».

Diverses appellations désignent ces squats : certains jeunes de FreshWota Wan se reconnaissent comme les Mi stap Ghetto, à FreshWota Foa, ce sont les Kingston 4, à Tebakor, où réside une large communauté de Man Tanna, il s'agit des TRK qui signifie Tebakor Raskel Kids (les jeunes voyous de Tebakor), à Nambatri ce sont les Kanaky, etc. Ces différents squats renvoient aux lieux d'habitation ou domiciles et sont connus de tous. Lorsqu'un problème survient, les protagonistes sont désignés selon leur quartier de résidence. L'appellation squat est récente, mais le phénomène de rassemblement des jeunes, montré sous une terminologie reconnue, existait déjà il y a une dizaine d'années. Un des plus célèbres groupes, les Vietnam Tu de Fresh Wota Tu est connu d'une large majorité de citadins actuels. Les nouvelles créations lexicales proviennent de ces jeunes urbains, souvent sans emploi, qui passent leur journée ensemble à " tuer le temps " selon l'expression en bichlamar déjà citée : kilim taem. La formation de ces groupes varie selon le sexe : les jeunes filles assujetties à maintes tâches domestiques (préparation des repas, ménage, lessive, garde des enfants, etc.) ne sont pas aussi libres de se déplacer que les garçons (Mitchell, 2004). Cette situation en milieu urbain est héritée d'une répartition sexuée des tâches (Jolly, 1994).

De nombreuses expressions voient le jour, certaines devenant rapidement plus populaires que d'autres et se diffusant dans la capitale, et parfois en zone rurale :

$$
\begin{aligned}
& \text { Fae } i \text { ded: "Je t'ai cloué le bec / cassé ». } \\
& \text { Yu save finis: "Tu vois quoi ». } \\
& \text { Onesti: "Je t'assure ». }
\end{aligned}
$$

D'autres expressions sont utilisées uniquement à Port-Vila ou à Luganville et très rarement dans les îles:

Nil blong koven : "Cigarette ", littéralement « clou de cercueil ».

Yu skrinim: "Tu captes », provient du mot skrin, de l'anglais screen, « écran ». 
En raison de la population relativement faible de la capitale (équivalente par exemple à celle d'une petite ville de province en France), il est parfois possible de remonter jusqu'aux initiateurs de certaines formulations, caractéristique soulignée par Crowley (1992). Le cas de l'expression stikim nek, très répandue aujourd'hui, illustre ce phénomène. Elle signifie "profiter de quelqu'un " et son origine est attribuée aux jeunes urbains de Fresh Wota Tu. L'image utilisée dans cette expression est celle du moustique qui suce le sang. À la fin des années 1990, les jeunes disaient Motin ${ }^{28}$ lorsque quelqu'un profitait d'une autre personne. Ensuite, le terme a évolué en stikim nek ${ }^{29}$, puis un glissement sémantique s'est produit et l'expression drink blod ${ }^{30}$ est apparue.

\section{Évolution du pidgin et processus d'identification}

Parallèlement au développement et à l'enrichissement du pidgin, le bichlamar connaît aussi un rapprochement très important avec la langue superstrat $^{31}$, l'anglais. Ce phénomène n'est cependant pas récent et avait déjà été constaté par Charpentier à la fin des années 1970 (Charpentier, 1979). Les opinions sont divergentes quant au continuum linguistique : certains spécialistes, comme Charpentier (1979), prévoyaient une "dépidginisation " du bichlamar et son rapprochement avec l'anglais. D'autres, comme Tryon (1987), pensaient que l'anglicisation du bichlamar allait rester un phénomène urbain et que les zones rurales allaient conserver leur différence. Selon nos propres observations, le bichlamar se décréolise partiellement en milieu urbain, au sein des populations éduquées ayant atteint le niveau scolaire de la douzième ou plus, ou ayant fait des études supérieures. Le lexique s'enrichit d'apports anglais : des termes utilisés en pidgin basilectal sont progressivement remplacés par des formes anglicisées. Autrefois, pour dire " vouloir " en bichlamar, wande était la forme employée, puis le mot a évolué en wantem, et aujourd'hui, wishim est de plus en plus utilisé. De même, pour dire "fermer ", klosem remplace progressivement sarem, ou pour dire « sentir (au sens de ressentir) ", harem devient filim; harem save se transforme en understandem pour dire « comprendre ». Et les exemples foisonnent. La forme grammaticale de la langue subit aussi une influence de la langue superstrat. Le bichlamar ne dispose pas de pluriel et le marqueur de pluralité « $s$ " ne s'entend pas. Pourtant de plus en plus, le « $s$ » s'immisce dans le pidgin oral. Désormais, on peut entendre :

$$
\begin{aligned}
& \text { Mi gat } 23 \text { yias : "J'ai } 23 \text { ans ». } \\
& \text { Mi kat tri brotas : "J'ai } 3 \text { frères ". }
\end{aligned}
$$

La forme écrite de la langue est particulièrement révélatrice de l'influence de l'anglais sur le pidgin. De plus en plus, dans les journaux, les mots dont l'orthographe est méconnue sont directement écrits en anglais :

\section{belif blo sospen [...] $i$ tekem aot attention blo mama [...] mi putum clay pot ia $i$ ko lo fire s. $^{32}$ "les croyances dans les casseroles [...] attirent l'attention des femmes [...], je mets cette poterie à cuire». Mi likem text ${ }^{33}$ : "J'aime envoyer des "textos" ".}

Cette anglicisation du pidgin écrit en l'absence d'enseignement du bichlamar dans les établissements scolaires est très fréquente. Cependant, certains organismes (Wan Smol Bag, Vanuatu Kaljoral Senta) ou journalistes (Gratien Mossoul) se réfèrent au dictionnaire de Crowley et écrivent le pidgin sous sa forme reconnue par le Conseil national des Langues du Vanuatu. Les médias participent aussi au rapprochement du pidgin et de l'anglais, comme la radio Capitol $107 \mathrm{fm}$, dont l'ensemble des animateurs a effectué ses études à l'étranger, principalement en Australie ou en Nouvelle-Zélande :

this dedication i stap go long... : " cette dédicace est so let's hit back to the tune afta hemia bae yumi kat meteo ${ }^{34}:$ "revenons maintenant à la musique et après nous écouterons la météo ».

Toutefois, il s'agit souvent pour des individus bilingues anglais/bichlamar d'une alternance de langues plus que d'une anglicisation, qui ne signifie pas forcément décréolisation, mais indique que les langues disposent d'un statut et d'un mode d'usage différents.

28. Motin est le nom bichlamar de la marque australienne d'insecticide Mortein, la plus répandue au Vanuatu.

29. Stikim nek se traduit par "sucer le sang" (littéralement "piquer le cou ").

30. Drink blod: "boire du sang ". Cette formule est devenue particulièrement populaire ; elle a été adoptée par des productions cinématographiques sur les vampires (Twilight) très appréciées du jeune public.

31. Un superstrat est, en linguistique, une langue qui en influence une autre sans toutefois la remplacer. Dans les phénomènes de pidginisation et de créolisation, les langues de contact s'inscrivent dans un continuum linguistique : le basilecte est la forme la plus proche - dans le temps et dans l'espace social - de la forme première du pidgin, le mésolecte représente une étape intermédiaire dans l'évolution du pidgin (pendant laquelle on observe l'introduction de morphème) ; et enfin l'acrolecte correspond à des formes très proches de la langue superstrat (Bickerton, 1975; Moreau, 1997).

32. Extrait de Lapita, de Esther Tinning in Storian, Vanuatu's Lifestyle Magazine 6, mai 2010.

33. Extrait d'une publicité pour la compagnie de téléphonie Digicel.

34. Les termes en gras indiquent les expressions anglicisées. Extrait de diverses émissions enregistrées respectivement les 2 mars et 13 avril 2010. 
De grandes disparités résident entre l'usage et les représentations des langues officielles et des langues vernaculaires. La langue est toujours utilisée comme un marqueur de positionnement social et, selon Bourdieu, les échanges et les interactions linguistiques sont autant $\mathrm{d}$ ' " agents " qui contribuent à reproduire la structure sociale. Cet auteur qualifie d' $«$ illusion du communisme linguistique " (1991:27) le travail des linguistes qui donnent l'impression qu'existe une langue entièrement similaire et commune aux individus d'une même communauté linguistique. Pour lui, le contexte socio-économique est indissociable de l'étude de la langue en elle-même et les dispositions linguistiques des individus sont perçues comme des " agents " qui poussent à agir et à réagir d'une certaine manière. Bourdieu applique son concept d'habitus à la linguistique dans son ouvrage de référence $C e$ que parler veut dire (1982). Il développe l'idée que l'État induit des relations de forces et de pouvoir entre les langues:

"C'est dans le processus de constitution de l'État que se créent les conditions de la constitution d'un marché linguistique unifié et dominé par la langue officielle (obligation dans les espaces publics officiels, dans l'éducation, etc.). " (Bourdieu, $2001: 30$ )

L'État fixe la norme sur le " marché linguistique ", instaurant ainsi dans des pays plurilingues $^{35}$ comme le Vanuatu des rapports de domination entre les idiomes. Dans le cas du Vanuatu, l'anglais et le français sont considérés comme des langues prestigieuses parce qu'elles sont écrites et enseignées ; elles permettent aux individus d'envisager leur promotion sociale (à travers le système éducatif et la maîtrise des langues). Les deux langues officielles sont valorisées consciemment ou inconsciemment par les individus. Le bichlamar, quant à lui, peine à bénéficier d'une image positive bien qu'il soit la langue nationale et que chacun reconnaisse l'utilité de cette langue de contact. Malgré le statut politiquement et symboliquement élevé du bichlamar, puisqu'il est inscrit comme langue nationale et officielle dans l'article 3 alinéa $1^{\text {er }}$ de la Constitution, le pidgin reste infériorisé par la classe dirigeante (des raisons historiques pré- et post- indépendance expliquent en partie l'instabilité du bichlamar). Lors du discours officiel annuel donné à la résidence de l'Ambassadeur de France le 14 juillet 2010, le président de la République du Vanuatu s'est exprimé en anglais (le président a fréquenté un établissement anglophone), laissant penser que le bichlamar n'est pas une langue suffisamment distinguée pour être parlée devant les institutions étrangères, de surcroît ex-puissances coloniales. En raison de la qualité de l'anglais prononcé et traduit en simultané, ce discours paraissait incongru ; un discours en bichlamar compris majoritairement par le public présent aurait été bienvenu et apprécié. Durant les sessions parlementaires, l'ensemble des débats s'effectuent en bichlamar mais les retranscriptions ne se font qu'en anglais - parfois en français faute des moyens techniques et financiers nécessaires aux traductions, selon le personnel ${ }^{36}$. Cette situation ambivalente rend complexe les représentations du bichlamar qui demeure la langue maternelle d'une partie de plus en plus large de la population urbaine. Pourtant, le phénomène de créolisation observée à Port-Vila confère au pidgin une nouvelle légitimité. Mais le processus de légitimation est lent car il faut du temps pour que les individus parviennent progressivement à intégrer de façon souvent semi-consciente leur attachement à une langue et à en être fiers.

L'éducation joue un rôle considérable dans la vision et l'appropriation du bichlamar. Les anglophones et les francophones ont une représentation et une utilisation différentes de cette langue. Proportionnellement, les francophones utilisent plus le français que les anglophones l'anglais (Charpentier, 1979 ; Siegel, 2008). Par ailleurs, les premiers conservent la structure du pidgin tout en y insérant du lexique français, en remplaçant un mot d'origine anglaise par sa traduction en français :

bae mi kakai glace: « je vais manger de la glace ", où le terme " glace " remplace aeskrim en bichlamar ;

mi wet lo wan formation blo traduire gud ol loi mo ol document ${ }^{37}:$ :j'attends une formation pour traduire les documents et les lois»

alors que le locuteur aurait dû dire :

mi wet lo wan trening blong translatem gud ol dokumen mo ol lao.

Chez les jeunes ayant une bonne connaissance d'une des deux langues d'enseignement, le bichlamar est une langue utilitaire et véhiculaire qui a peu d'importance identitaire. Souvent, son utilisation renvoie au domaine de la vie quotidienne ordinaire. Au lycée français de Port-Vila, établissement conventionné par l'Agence pour l'enseignement du français à l'étranger (AEFE), où sont scolarisés un grand nombre d'enfants de l'élite francophone, le bichlamar est perçu par

35. Claude Hadège désigne un État où s'enseignent et se parlent plusieurs langues comme plurilingue. Il propose d'appeler multilingue non plus un État mais un individu qui parle deux ou trois langues.

36. Entretien avec Lydia, traductrice anglophone, durant les sessions parlementaires, le 18 mars 2011.

37. Extrait d'un entretien avec une personne ayant effectué une première année de Lettres modernes à l'Université de Nouvelle-Calédonie (Nouméa) et travaillant au bureau du Premier ministre comme traducteur anglais/français et bichlamar/français. 
certains élèves comme une "fausse " langue ${ }^{38}$. L'ensemble des lycéens, majoritairement des métis, qui prétendent ne pas attacher d'importance identitaire au bichlamar, ont tous pour première langue le français, l'anglais ou une autre langue internationale. En revanche, les $\mathrm{Ni}$-Vanuatu qui ont pour langue maternelle une langue vernaculaire du Vanuatu, reconnaissent le bichlamar comme une langue faisant partie de leur identité culturelle. En maternelle, le bichlamar est la langue de communication de tous les enfants en début d'année; en primaire, il s'agit de la langue des jeux de cour de récréation entre les élèves; de même, au lycée et au collège, le bichlamar est l'idiome le plus répandu en dehors de la classe. Lorigine sociale des individus nous semble être déterminante dans la reconnaissance qu'ils accordent au pidgin. Ceux qui sont peu scolarisés (ayant arrêté leurs études au collège ou en primaire) paraissent parfois honteux de ne parler que le bichlamar, mais assument toutefois mieux leur usage du pidgin et se revendiquent défenseurs de cette langue :

bislama hemi nasonal lanwis blong yumi, mas toktok bislama nomo : "le bichlamar est la langue nationale, il faut parler seulement bichlamar ».

La créolisation du pidgin en milieu urbain a renforcé et rendu son usage et la position de ses défenseurs légitimes.

\section{Conclusion}

Trente ans après l'indépendance de la nation, attiré par les lumières de l'Occident tout en revendiquant ses spécificités culturelles et linguistiques, le Vanuatu doit faire face à une accélération des changements engagés. Dans ce contexte, pour des raisons socio-historiques, le bichlamar apparaît comme un outil incontournable pour en mesurer l'ampleur. L'exode rural des années 1970 a entraîné de profonds bouleversements dans la société urbaine de Port-Vila, avec l'apparition de rapports de classes de plus en plus marqués, qui prennent parfois des formes inédites chez les jeunes citadins en manque de repères et de perspectives d'avenir. Avec l'émergence d'une classe moyenne, une société de consommation s'est développée, à laquelle est associé un nouveau mode de vie. Les médias, la publicité, le développement des transports et des télécommunications concourent à rétrécir l'espace et à désenclaver les populations isolées. Ces changements se reflètent parfaitement dans la diffusion du bichlamar qui, progressivement, s'immisce dans les langues vernaculaires et de- vient une référence pour les jeunes, même en zone rurale. Nous n'avons pas abordé dans cet article la jeunesse des îles mais ses relations au bichlamar sont tout aussi intéressantes qu'en milieu urbain, avec, à nouveau, une profonde et nouvelle appropriation au pidgin. Nous pourrons émettre de nouvelles hypothèses après avoir mené une étude de la langue employée par la jeunesse qui fréquentera le TKS, Tafea Kaljoral Senta et sa TAFEA Kastom Digitol Laebri (projet coordonné par Jacob Kapere et Marc Tabani), premier centre culturel d'envergure qui vient d'ouvrir ses portes hors de la capitale, à Tanna, et dont la majeure partie des activités est destinée aux jeunes. Des organisations comme le Yut Senta ou le Vanuatu Young People Project incarnent les nouvelles aspirations et problèmes des jeunes urbains, génération charnière, car il s'agit de la génération (la seconde ayant entièrement grandi à Port-Vila) qui installe véritablement l' " urbanité » comme mode de vie, en expérimentant de nouvelles réalités. Le bichlamar légitimise sa place dans son nouveau statut de créole, pour un nombre croissant de citadins, mais il reste néanmoins une langue au statut ambigu pour la classe dirigeante.

L'éducation semble donc être un moyen essentiel pour faire évoluer la vision péjorative associée au pidgin et redonner ainsi une image positive à ses locuteurs. En 2009, le ministère de l'Éducation a proposé une réforme de sa politique linguistique éducative qui est actuellement en cours d'évaluation. Plusieurs scénarios sont envisagés dont celui où le bichlamar deviendrait une langue enseignée ou une langue d'enseignement dans les zones urbaines à forte mixité linguistique. Certes, un tel scénario soulève les difficultés du manque de stabilité de la langue, mais cette démarche permettrait de légitimer réellement - et non plus uniquement symboliquement - le bichlamar. De plus, intégrer ce pidgin au sein du système éducatif garantirait sa survie (bien que rien ne laisse penser qu'il s'éteigne), clarifierait sa position et le différencierait selon nous de la langue superstrat, l'anglais. Pour l'heure, trente ans après l'élévation du pidgin au rang de langue officielle et nationale du Vanuatu, le bichlamar incarne l'accentuation des inégalités sociales de l'archipel plus qu'il ne les estompe tout en unifiant les $\mathrm{Ni}$-Vanuatu.

\section{BIBLIOGRAPHIE}

Bickerton Derek, 1975. Dynamic of a creole system, Cambridge, University Press.

Bonnemaison Joël, 1974. Changement dans la vie rurale et mutation migratoire aux Nou- 
velles Hébrides, Cahier de l'ORSTOM série Sciences humaines 11(2), pp. 259-286.

-, 1977. Système de migrations, croissance urbaine à Port-Vila et Luganville, Paris, OrsTOM, Travaux et Documents.

Bourdieu Pierre, 1991. Langage et pouvoir symbolique, Paris, Seuil.

Calvet Louis-Jean, 2002. Le marché aux langues. Les effets linguistiques de la mondialisation, Paris, Plon.

Charpentier Jean Michel, 1979. Le pidgin bislama (n) et le multilinguisme aux Nouvelles Hébrides, Paris, SELAF.

-, 1982. Quand et où parler de bilinguisme et de diglossie ? Le problème des pidgins et des patois assimilés dans le cas du bichelamar de Vanuatu (ex-Nouvelles-Hébrides) et du patois du Poitou, La linguistique 18 (1), pp. 65-84.

—, 1998. Sabirs, Pidgins, Créoles. Les langues de contact dans le Pacifique, in A. Bensa et J.-C. Rivierre (éds), Le Pacifique, un monde épars, Paris, L'Harmattan, pp. 106-125.

Charpentier Jean-Michel et Darrell Tryon, 1982. Functions of Bislama in the New Hebrides and independent Vanuatu, English World-Wide 3 (2), pp. 146-160.

Crowley Terry, 1975. A new Bislama Dictionary, Suva, Institut of Pacific Studies.

-, 1990. Beach-la-MAR To Bislama, the emergence of a National Language in Vanuatu, Oxford, Clarendon Press.

- , 1995. The national drink and the national language in Vanuatu, Journal of Polynesian Society 104 (1), pp. 7-22.

-, 2000, Vernaculars in education in Vanuatu, Report prepared for World Bank and Vanuatu Ministry of Education, Youth and Sport, Port-Vila, Vanuatu.

Hagège Claude, 1996. L'enfant aux deux langues, Paris, Odile Jacob.

Joluy Margaret, 1994. Women of the Place: Kastom, Colonialism and Gender in Vanuatu, Amsterdam, Harwood Academic Publishers.

Martinet André, 1996. Eléments de linguistique générale, Paris, Armand Colin.
Mrtchell Jean, 2004. Killing Time in a Postcolonial Town: Young People and Settlements in Port-Vila, Vanuatu, in V. Lockwood (ed.), Globalisation and culture change in the Pacific Islands, New-York, Prentice Hall, pp. 358-376.

Moreau Marie-Louise, 1997. Superstrat, Sociolinguistique. Concepts de base, Mardaga, p. 282.

Moyse-Faurie Claire, 2000. Langues minoritaires et politiques linguistiques : le cas des langues océaniennes, Les langues en danger, Paris, Société de Linguistique, Mémoires 8, pp. 79-104.

Philibert Jean-Marc, 1992. Social Change in Vanuatu, in A. Robillard (ed.), Social Change in the Pacific Island, London, Keagan Paul, pp. 98-133.

SIEGEL Jeff, 2008. The Emergence of pidgin and Creole Languages, Oxford, Oxford University Press.

SimÉONi Patricia (éd.), 2009. Atlas du Vanouatou (Vanuatu), Port-Vila, éditions Géo-Consulte.

Stern Monika, 2002. Les femmes, les nattes et la musique sur l'île de Pentecôte (Vanuatu), thèse de doctorat, Paris, Université Sorbonne IV.

TABANi Marc, 2002. Les pouvoirs de la coutume à Vanuatu. Traditionalisme et édification nationale, Paris, L'Harmattan.

TRYON Darrell, 1987. Bislama, an introduction to the national language of Vanuatu, Canberra, The Australian National University.

Tryon Darrell et Jean-Michel Charpentier, 2004. Trends in Linguistics, Pacific Pidgins and Creoles Origins, Growth and Developpement, Berlin and New York, Mouton de Gruyter.

Vanuatu National Statistics Office, 2009. National Census of Population and Housing, Port Vila, Ministry of Finance and Economic Management.

VANDEPutte Tavo Leslie, 2011 (à paraître). Quand la publicité fait son apparition : éléments d'analyse ethnolinguistique des deux compagnies de télécommunication du Vanuatu, in R.G. Zimmer (éd.), Limage en Mélanésie : acte du colloque CORAIL 2010, Paris, L'Harmattan. 